\title{
A Pharmacological Strategy Using Stemofoline for more Efficacious Chemotherapeutic Treatments Against Human Multidrug Resistant Leukemic Cells
}

\author{
Sonthaya Umsumarng ${ }^{1,2}$, Sariya Mapoung ${ }^{1,2}$, Supachai Yodkeeree ${ }^{1,2}$, Stephen G \\ Pyne $^{3}$, Pornngarm Limtrakul Dejkriengkraikul ${ }^{1,2 *}$
}

\begin{abstract}
Our previous study reported that stemofoline (STF) exhibited a synergistic effect with chemotherapeutic drugs in human multidrug-resistant (MDR) leukemic cells (K526/Adr) by inhibiting the function of P-glycoprotein, which is a membrane transporter that is overexpressed in several types of MDR cancers. This study further investigated the effects of a combination treatment of STF and doxorubicin (DOX) in vitro and in vivo. The combination treatment of $50 \mathrm{mg} / \mathrm{kg}$ of STF strongly enhanced the anti-tumor activity of DOX in SCID-beige mice bearing K562/Adr xenografts without additional toxicity when compared to the single treatment groups. Additionally, an examination of the proliferation markers (Ki67) and the apoptotic marker (TUNEL) in tumor tissues in each group revealed that the combination therapy significantly reduced Ki67 positive cells and increased apoptotic cells. From the in vitro experiments we also found that this combination treatment dramatically induced $\mathrm{G}_{1}$ and $\mathrm{G}_{2} \mathrm{M}$ arrest in $\mathrm{K} 562 / \mathrm{Adr}$ when compared to a single treatment of DOX. STF treatment alone did not show any cytotoxic effect to the cells. These results suggest that the accumulation of DOX enhanced by STF was sufficient to induce cell cycle arrest in K562/Adr. These findings support our previous in vitro data and indicate the possibility of developing STF as an adjuvant therapy in cancer treatments.
\end{abstract}

Keywords: Chemotherapy- Leukemia- P-glycoprotein- Xenograft model- Stemofoline

Asian Pac J Cancer Prev, 19 (12), 3533-3543

\section{Introduction}

Leukemia is a cancer that affects the blood and bone marrow, the spongy center of bones where blood cells are formed. Leukemia treatment usually involves chemotherapy, bone morrow implantation and radiotherapy (Baruchel et al., 2009; Matsumura, 2009). Chronic myeloid leukemia (CML) is a myeloproliferative disorder of hemopoitic stem cells. The incident rate of CML in males has been reported to be higher than the rate for females (male/female ratio is 1.3-1.5:1) (Quintas-Cardama and Cortes, 2006; Mendizabal et al., 2013). There has been some improvement in survival rates since the introduction of therapeutic drugs including the anthracyclines (doxorubicin, epirubicin), the vinca alkaloids (vinblastine, vincristine) and methotrexate, L-asparaginase and alkylating agents (Norgaard et al., 2004). Chemotherapy for CML treatment uses one or usually at least two of the above-mentioned chemical substances. Such treatments not only destroy or reduce tumor cell growth but also increase the 5-year survival rates for cancer patient. However, resistance to anti-neoplastic drugs can develop in several types of cancer patients who have taken chemotherapy over long periods of time (Tiwari et al., 2011; Binkhathlan and Lavasanifar, 2013).

Chemo-resistance or multidrug resistance (MDR) is a major problem in cancer treatments. The various mechanisms of MDR include overexpression of drug transporters, an increase in detoxification mechanisms along with an induction of DNA repairing enzymes, and altered drug targets (deletion, translocation and amplification of drug target) (Gottesman et al., 2002; Fojo and Menefee, 2007; Lage, 2008). The causes of death in leukemic patients are infection, loss of muscle control and heart failure. However, several studies have described the increased P-gp expression after the initial chemotherapy treatment that has repeatedly been linked to poor outcomes in some forms of leukemia (Marie et al., 1996; Leonard et al., 2003). Doxorubicin (DOX) is an effective form of chemotherapy against both hematological malignancy and solid tumors. DOX exhibited higher efficacy on leukemic patient by reduction of blast cells in blood circulation. Anti-tumor mechanisms of DOX are achieved by the induction of free radicals, which are linked to DNA

${ }^{1}$ Department of Biochemistry, Faculty of Medicine, ${ }^{2}$ Excellent Center for Research and Development of Natural Products for Health, Chiang Mai University, Chiang Mai, Thailand, ${ }^{3}$ School of Chemistry, University of Wollongong, Wollongong, New South Wales, Australia.*For Correspondence: pornngarm.d@cmu.ac.th 
damage, and inhibition of DNA and RNA synthesis by inhibition of topoisomerase II (Rabbani et al., 2005; Rogalska et al., 2008; Szwed et al., 2016). However, DOX has been shown to give rise to the development of drug resistance, which might be due to an increase in P-glycoprotein (P-gp) expression (de Moraes et al., 2013).

Many P-gp inhibitors have been reported, such as the first generation drugs verapamil, quinidine and cyclosporine $\mathrm{A}$, and the second generation drugs dexverapamil, emopamil and PSC 833. Therefore, researchers have tried to identify the third-generation of P-gp inhibitors (with prolonged half-life and reduction of toxicity) from plants or natural products. These agents are currently being studied in clinical trials (Krishna and Mayer, 2000; Leonard et al., 2003). We have focused on natural compounds as inhibitors of P-gp to enhance the efficacy of anticancer drugs against MDR cancer cells. Many natural product P-gp inhibitors have been reported, including curcumin (Anuchapreeda et al., 2006; Limtrakul et al., 2007), kuguacin J (Pitchakarn et al., 2012) and calidonine (El-Readi et al., 2013).

Stemona plants that are found in East and South-east Asia are important herbal plants with various medicinal and pharmacological properties. Plant extracts obtained from Stemona tubulosa, S. sessilifolia and S. japonica have been reported for their antitrussive activities in mouse model studies ( $\mathrm{Hu}$ et al., 2009). The S. tubulosa extract has been reported to have an anti-inflammatory effect of the lungs in cigarette smoke-induced pulmonary inflammation both in vitro and in vivo (Lee et al., 2014; Lim et al., 2015). The S. tubulosa and S.collinsae extracts have been reported to have anti-proliferative effects on the medullary thyroid carcinoma cell line (Li et al., 2007). However, the activity of Stemona plant extracts against drug resistant cancer cells has not yet been reported. In our previous studies we reported that the extract of Stemona burkillii could enhance the chemotherapeutic sensitivity in human MDR cervical carcinoma (KB-V1) cells (Chanmahasathien et al., 2011a; Pyne SG et al., 2017). We then purified and identified the active compound identified as the Stemona alkaloid stemofoline (STF).

In our previous studies we found that STF showed significant synergistic effects with conventional anticancer agents against KB-V1 and human MDR leukemic cells (K526/Adr). STF inhibited P-gp function leading to increases of P-gp fluorescent substrates (calcein-AM and rhodamine 123) and [14C]-DOX intracellular accumulation in KB-V1 and K562/Adr cells (Chanmahasathien et al., 2011a; Umsumarng et al., 2013; Umsumarng et al., 2015). The biochemical mechanism of STF could be that it reverses the MDR cancer phenotype by inhibition of P-gp function via direct interaction with the substrate binding domain but by without affecting P-gp expression (Chanmahasathien et al., 2011b). However, the MDR reversal property of STF in an animal model has not yet been demonstrated. In this study, we further investigated the effect of the combination treatment of STF and DOX in an animal model. We first investigated the acute toxicity of STF by acute toxicity testing and studied the MDR modulating property of STF in MDR leukemic cells in a xenograft model. Next, we determined the mechanism for synergistic effect of STF and DOX in MDR leukemic cells in vitro.

\section{Materials and Methods}

\section{Stemofoline extraction and isolation}

STF was isolated from Stemona burkillii root extract and its structure is shown in Figure 1. Dried root powders were extracted with a solution of $95 \%$ of ethanol three times at room temperature. The ethanolic extract was combined and evaporated using a rotary evaporator. STF was purified as previously described (Sastraruji et al., 2010; Chanmahasathien et al., 2011a; Sastraruji et al., 2012).

\section{Cells and cell culture}

K562/Adr (human MDR leukemic cell line, CML) was purchased from RIKEN Cell Bank (Tsukuba, Ibaraki, Japan). K562 (human drug sensitive leukemic cell line) was purchased from The American type Culture Collection (ATCC, Manassas, VA, U.S.A.). Both cell lines were maintained and cultured in a $37^{\circ} \mathrm{C}$ humidified atmosphere comprised of 5\% $\mathrm{CO}_{2}$ in RPMI-1640 (Nissui Pharmaceutical Co., Ltd., Taito-ku, Tokyo, Japan) with $10 \%$ fetal bovine serum (FBS), $5 \mathrm{~mm}$ L-glutamine, 50 $\mathrm{IU} / \mathrm{mL}$ penicillin and $50 \mathrm{~g} / \mathrm{mL}$ streptomycin. When the cells reached $70-80 \%$ confluence, they were harvested and plated either for subsequent passages or for drug treatments. K562/Adr cells were routinely maintained in RPMI-1640 culture medium containing $700 \mathrm{nM}$ of doxorubicin (DOX, Sigma Chemical Co., St. Louis, MO, U.S.A.) and grown in a drug-free medium for at least two days before the experiment.

\section{Animals}

All animal experiments were performed under protocols approved by the Committee of Animal Care and Use of Saitama University. Eight-week-old male ICR mice and 6-week-old male SCID-Beige mice were purchased from Charles River Japan, Inc. (Atsugi, Japan) and housed in plastic cages with bedding consisting of hardwood chips in an air-conditioned room at $23 \pm 2^{\circ} \mathrm{C}$ and at $55 \pm 5 \%$ humidity with a $12 \mathrm{~h}$ light/dark cycle. Diet and autoclaved water were available ad libitum.

\section{Acute toxicity testing}

The acute toxicity of STF was evaluated using eight-week-old 5 male ICR mice. The ICR mice were intraperitoneally (i.p.) injected with STF ( $50 \mathrm{mg} / \mathrm{kg} \mathrm{BW})$ according to OECD 420 guidelines. Mice morbidity and mortality were recorded daily. After treatment for two weeks, the surviving mice were sacrificed and $\mathrm{LD}_{50}$ values were calculated. Mice serum samples were collected to investigate liver function by determining alanine aminotransferase (ALT) and aspartate aminotransferase (AST) levels. Renal function was tested by determination of blood urea nitrogen (BUN) and creatinine levels. Internal organs (liver, spleen, heart, lungs and kidneys) were measured and gross examination was observed on abnormal lesions. Consequently, sections of the internal organs were fixed in 10\% of formaldehyde in PBS. These 
sections of internal organs were prepared for paraffin embedding. The paraffin sections were then stained by $\mathrm{H}$ and $\mathrm{E}$. Pathological evaluation was conducted by a Veterinarian Pathologist. The acute toxicity was determined by mice body weight, mice survival rate, gross examination and histopathological examination of internal organs and the data were compared with the references values.

\section{Effect of STF on DOX sensitivity in a K562/Adr xenograft model}

Human multidrug resistant leukemic cell (K562/ Adr) xenograft model was used for an investigation of the MDR reversing property of STF on DOX sensitivity. Six-week-old male SCID-beige mice (20 mice) were implanted with K652/Adr cells $\left(5.0 \times 10^{6}\right.$ cells $)$ subcutaneously at the flank area of the mice (Beider et al., 2014). The tumors on the flank area of the mice were palpated every day. As soon as the tumors reached $100-200 \mathrm{~mm}^{3}$ in volume, the mice were divided into 4 groups: 1) vehicle control; $0.9 \% \mathrm{NaCl}$; IP 2) STF $50 \mathrm{mg} /$ $\mathrm{kg}$ BW; IP, 3) DOX $2 \mathrm{mg} / \mathrm{kg} \mathrm{BW}$; IV and 4) DOX $2 \mathrm{mg} /$ $\mathrm{kg}$ BW; IV plus STF $50 \mathrm{mg} / \mathrm{kg}$ BW; IP, respectively. For the combination treatment group, $50 \mathrm{mg} / \mathrm{kg} \mathrm{BW}$ of STF was administered for 30 minutes before DOX was applied. These treatments were applied every 4 days for 16 days. The mice that bore tumors were measured every 3 days and their weights were measured every 4 days. After which, all mice were sacrificed and primary tumors were removed and measured in terms of weight and volume (formula; axis $1 \mathrm{x}$ axis $2 \mathrm{x}$ axis 3 ) $\mathrm{x} 0.52$ (Pitchakarn et al., 2012; Punfa et al., 2014). Internal organs (liver, spleen, heart, lungs and kidneys) were inspected for abnormal lesions. Sections of the primary tumors and internal organs were then fixed in $10 \%$ of formaldehyde in PBS. These sections of the internal organs were used to prepare paraffin embeds. Paraffin sections were stained with $\mathrm{H}$ and $\mathrm{E}$. Pathological evaluations were conducted by Veterinarian Pathologist. Tumor sections were processed for $\mathrm{H}$ and $\mathrm{E}, \mathrm{Ki} 67$ immunohistochemistry staining and terminal deoxynucleotidyl transferase mediated dUTP nick end labeling (TUNEL) assay.

\section{Immunohistochemistry}

For Ki67 immunostaining, paraffin-embedded specimens were sectioned $(5 \mu \mathrm{m})$ and stained with Ki67 antibody (clone SP6) (Acris Antibodies GmbH, Herford, Germany) and then with anti-rabbit secondary antibody and avidin-biotin complex (Vectastatin Elite ABC kit; Vector Laboratory, Burlingame, CA). After which, the binding sites were visualized with diamino-benzidine. The sections were then counterstained lightly with hematoxylin for microscopic examination.

\section{TUNEL assay}

Apoptotic index values were determined by TUNEL assay. The assay was performed using ApopTag ${ }^{\circledR}$ Peroxidase In Situ Apoptosis Detection Kit (Merck millipore Billerica, Massachusetts, USA).

\section{Chemo-sensitivity testing by MTT colorimetric assay}

Cell viability of each leukemic cell line was measured using the MTT assay. Drug sensitive human leukemic cells (K562) and human MDR leukemic cells (K562/Adr) were plated in a 96-well-plate at a concentration $2.0 \times 10^{3}$ cells/well. After $24 \mathrm{~h}$, the cells were treated with $0-40 \mu \mathrm{M}$ of DOX with or without $5 \mu \mathrm{M}$ of STF. Cell lines were incubated under a temperature of $37^{\circ} \mathrm{C}$ at $5 \%$ of $\mathrm{CO}_{2}$. After 48 hours of treatment, 3-(4,5-dimethylthiazol-2-yl)2,5-diphenyltetrazolium bromide (MTT, Sigma Chemical Co., St. Louis, MO, U.S.A.) was added to each well for 4h. RPMI-1640 medium was then removed and $200 \mu \mathrm{L}$ of DMSO (Wako Pure Chemical Ind., Ltd., Chuo-ku, Osaka, Japan) was added to the specimen. Each leukemic cell line was incubated at $37^{\circ} \mathrm{C}, 5 \% \mathrm{CO}_{2}$ for $4 \mathrm{~h}$. Cell viability was assessed by determining the mean of MTT colorimetric assay. In each experiment, determinations were carried out in triplicate. $\mathrm{IC}_{50}$ value of each cell line was calculated.

Apoptosis analysis by annexin $V$ and propidium iodine (PI) staining

$\mathrm{K} 562 / \mathrm{Adr}$ cells were plated in a 6-well plate at a concentration of $5.0 \times 10^{5}$ cells/well. After $24 \mathrm{~h}$, the cells were treated with various concentrations of DOX (0-5 $\mu \mathrm{M})$ in the absence or presence of $5 \mu \mathrm{M}$ of STF for $48 \mathrm{~h}$. Cells were then collected and washed twice with ice-cold PBS. The cell line was stained with annexin V-FITC and PI according to the manufacturer's instructions using the annexin V-FITC apoptosis detection kit (BioVision Inc., Milpitas, CA, U.S.A.). Apoptotic cells were detected by flow cytometry. Annexin V-positive cells were considered indicative of early apoptosis. Both annexin $\mathrm{V}$ and PI-positive cells were considered indicative of late apoptosis.

\section{Cell cycle analysis by propidium iodine (PI) staining}

K562/Adr cells were plated in a 6-well-plate at a concentration of $5.0 \times 10^{5}$ cells/well. After $24 \mathrm{hrs}$, the cells were treated with various concentrations of DOX $(0-5 \mu \mathrm{M})$ with or without $5 \mu \mathrm{M}$ of STF for 48 hours. Leukemic cells were collected and washed twice with ice-cold PBS. The cell line was fixed with $70 \%$ cold ethanol for 30 minutes and incubated for 30 minutes by $0.25 \mathrm{mg} / \mathrm{mL}$ of RNase $\mathrm{A}$ in $1.12 \%$ of sodium citrate buffer. Finally, the cell line was stained with PI for 30 minutes. Cell cycling was performed by flow cytometry.

\section{Statistical analysis}

All in vitro experiments were performed in at least triplicate to confirm reproducibility. Statistical analyses were performed with mean \pm S.D. values using one-way ANOVA, the Bonferroni correction and Dunnett's test. Statistical significance was determined at $p<0.05$, $p<0.01$ or $p<0.001$.

\section{Results}

\section{Acute toxicity study of STF}

The acute toxicity study of STF was evaluated using five eight-week-old male ICR mice. The ICR mice were i.p. injected with highest solubility of STF $(50 \mathrm{mg} / \mathrm{kg}$

Asian Pacific Journal of Cancer Prevention, Vol $19 \mathbf{3 5 3 5}$ 
Table 1. Effect of STF on Acute Toxicity in Animal Models

\begin{tabular}{lccccccc}
\hline Parameters & Mouse\#1 & Mouse\#2 & Mouse\#3 & Mouse\#4 & Mouse\#5 & Mean \pm SD & Ref. \\
\hline Mice BW (g) (start) & 33.5 & 31.88 & 32.4 & 34.1 & 33.8 & $33.14 \pm 0.95$ & $30.50 \pm 2.60^{\mathrm{a}}$ \\
Mice BW (g) (end) & 31.62 & 32.62 & 32.51 & 33.06 & 33.1 & $32.58 \pm 0.60$ & $32.50 \pm 3.40^{\mathrm{a}}$ \\
Survival rate (\%) & 100 & 100 & 100 & 100 & 100 & $100.00 \pm 0.00$ & - \\
Liver (g) & 2 & 1.84 & 1.95 & 2.01 & 2.05 & $1.97 \pm 0.08$ & $1.878 \pm 0.248^{\mathrm{a}}$ \\
Spleen (g) & 0.14 & 0.13 & 0.13 & 0.14 & 0.16 & $0.14 \pm 0.01$ & $0.145 \pm 0.021^{\mathrm{a}}$ \\
Heart (g) & 0.22 & 0.23 & 0.23 & 0.24 & 0.24 & $0.23 \pm 0.01$ & $0.175 \pm 0.023^{\mathrm{a}}$ \\
Rt. Kidney (g) & 0.4 & 0.37 & 0.43 & 0.39 & 0.44 & $0.41 \pm 0.03$ & $0.441 \pm 0.062^{\mathrm{a}}$ \\
Lt. Kidney (g) & 0.4 & 0.31 & 0.41 & 0.35 & 0.4 & $0.37 \pm 0.04$ & \\
ALT (U/L) & 15 & 15 & 20 & 13 & 14 & $15.40 \pm 2.70$ & $37.0 \pm 9.10^{\mathrm{b}}$ \\
AST (U/L) & 124 & 90 & 60 & 94 & 83 & $90.20 \pm 23.03$ & $100.00 \pm 14.60^{\mathrm{b}}$ \\
BUN (mg/dL) & 26 & 27 & 26 & 30 & 28 & $27.40 \pm 1.67$ & $28.54 \pm 3.16^{\mathrm{b}}$ \\
Creatinine (mg/dL) & 0.3 & 0.3 & 0.3 & 0.3 & 0.3 & $0.30 \pm 0.00$ & $0.43 \pm 0.18^{\mathrm{b}}$ \\
\hline ALT, Al
\end{tabular}

ALT, Alanine aminotransferase; AST, Aspartate aminotransferase; BUN, Blood urea nitrogen; aCharles River Laboratories Tech. 1986; ${ }^{b}$ Laurie el al., 2003 .

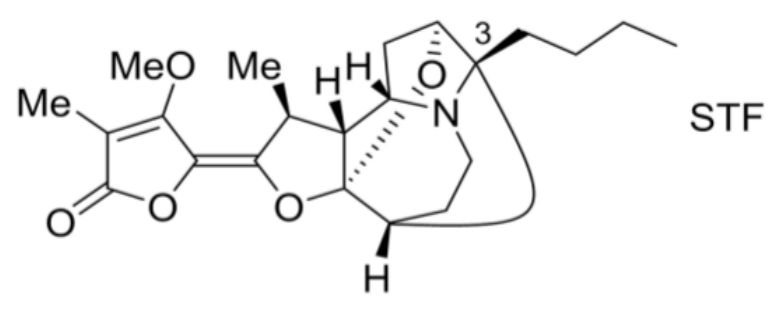

Figure 1. Structure of STF

BW) for 2 weeks. Our result shown that all mice were not observed acute toxicity. There were no differences in the body weight and internal organ weight when compared to the reference materials (Table 1).

STF-enhanced sensitivity of MDR leukemic cells to chemotherapeutic drug in vivo

Our results found that co-administration of $\mathrm{DOX}+\mathrm{STF}$ reduced the tumor volume of K562/Adr cells implanted in the back area of SCID-Beige mice when compared administration with the control $(p=0.3827)$, with STF $(p=0.1371)$ and with DOX $(p=0.4091)$ (Figure 2A and B). Moreover, tumor weight also significant reduced when the mice was co-administration with $\mathrm{DOX}+\mathrm{STF}$ when compare with control, STF and DOX (Figure 2C). An adverse effect on the body weight (Figure 2D) and internal organs (Table 2) of the host SCID-Beige mice was not detected. In addition, there is no histological lesion was observed in $\mathrm{H}$ and $\mathrm{E}$ staining of liver, spleen, heart, lung and kidney of all the treatment mice (Figure 2E).

$\mathrm{H}$ and $\mathrm{E}, \mathrm{Ki} 67$ and TUNEL staining are shown in Figure 3A. The Ki67 labeling index value was significantly reduced in the combination treatment group (Figure 3B) and TUNEL-positive cells of the transplanted tumor tissues were shown to have significantly increased in the combination treatment group (Figure 3C). These results could suggest that the treatment of STF and DOX revealed a significant synergistic effect by reducing cell proliferation and inducing apoptosis in K562/Adr transplantation SCID-beige mice.

STF-enhanced sensitivity of MDR leukemic cells to chemotherapeutic drug

We examined the MDR reversing property of STF and DOX co-administration in K562/Adr (MDR leukemic cells) and K562 (drug sensitive cells) cell lines. We found that co-administration of STF and DOX significantly induced growth inhibition in a dose dependent manner when compared with DOX single treatment in K562/ Adr $(p<0.001)$. This modulating effect was not found with a similar administration of DOX and STF to K562 cells (Figure 4A and B). Moreover, we demonstrated that treatment K562 and K562/ADR with STF $50 \mu \mathrm{M}$ for $48 \mathrm{~h}$ were not show cytotoxic to K562 and K562/Adr cells

Table 2. Effect of Treatment on the Body Weight and Internal Organs of Host SCID-Beige Mice

\begin{tabular}{lcccc}
\hline Parameters & Vehicle control group & STF treated group & DOX treated group & DOX+STF treated group \\
\hline Mice BW (g) (start) & $24.10 \pm 1.35$ & $24.03 \pm 1.16$ & $25.97 \pm 1.06$ & $24.23 \pm 1.58$ \\
Mice BW (g) (end) & $22.20 \pm 1.84$ & $22.73 \pm 2.32$ & $21.87 \pm 1.62$ & $20.83 \pm 1.97$ \\
Survival rate (\%) & 100 & 100 & 100 & 100 \\
Liver (g) & $1.03 \pm 0.15$ & $1.22 \pm 0.16$ & $1.08 \pm 0.27$ & $1.07 \pm 0.12$ \\
Spleen (g) & $0.04 \pm 0.02$ & $0.05 \pm 0.01$ & $0.03 \pm 0.01$ & $0.02 \pm 0.01$ \\
Heart (g) & $0.13 \pm 0.02$ & $0.13 \pm 0.02$ & $0.15 \pm 0.05$ & $0.10 \pm 0.03$ \\
Rt. Kidney (g) & $0.24 \pm 0.02$ & $0.23 \pm 0.05$ & $0.20 \pm 0.02$ & $0.17 \pm 0.03$ \\
Lt. Kidney (g) & $0.18 \pm 0.04$ & $0.23 \pm 0.07$ & $0.19 \pm 0.02$ & $0.19 \pm 0.02$ \\
Tumors weight (g) & $1.7 \pm 0.03$ & $2.05 \pm 0.67$ & $2.03 \pm 0.09$ & $0.99 \pm 0.17$ \\
\hline
\end{tabular}


(A)

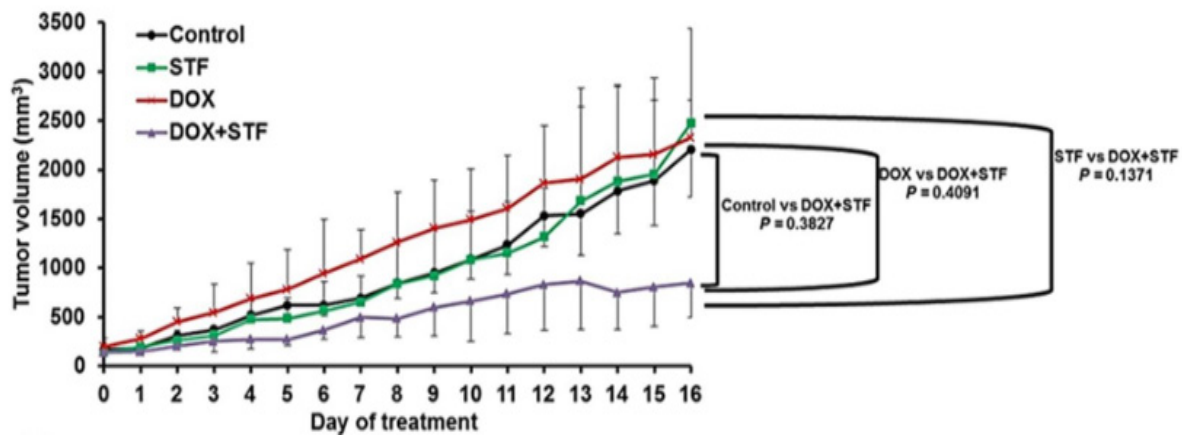

(B)

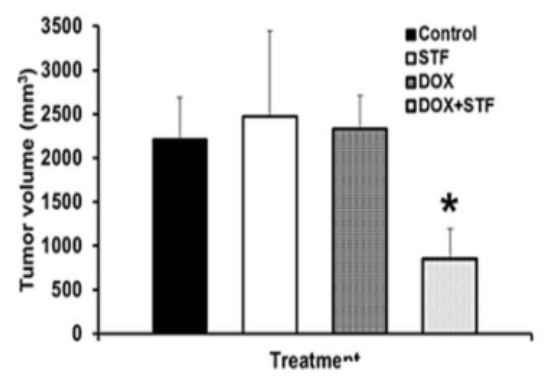

(C)

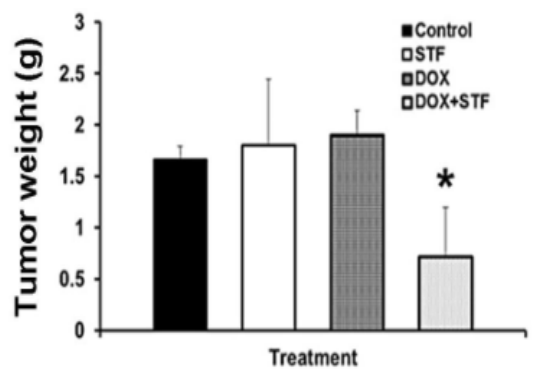

(D)

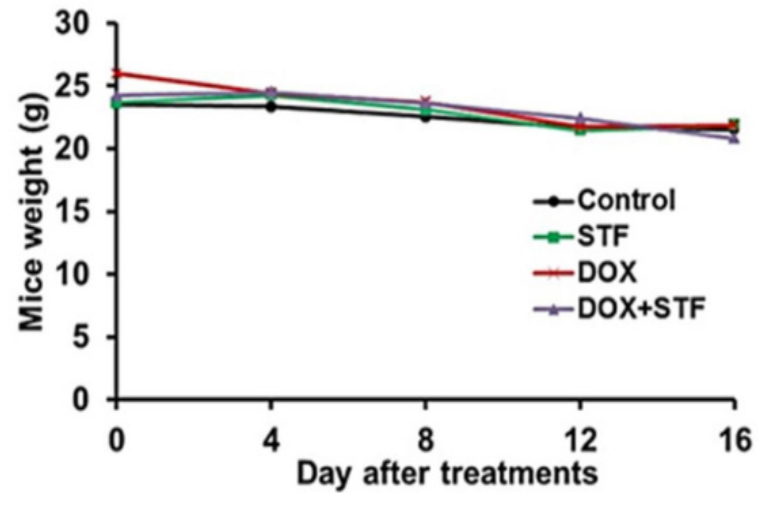

(E)

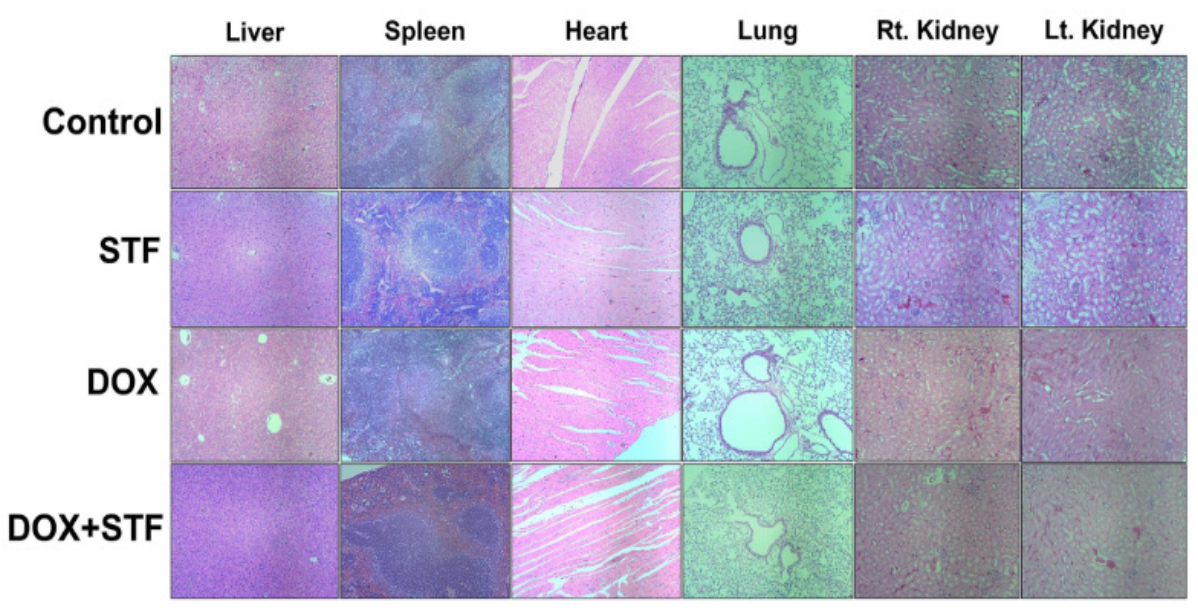

Figure 2. Potentiation of Antitumor Effects of DOX by STF in K562/Adr Xenograft Model. Mice were treated with saline (control); STF alone at $50 \mathrm{mg} / \mathrm{kg}$; DOX alone at $2 \mathrm{mg} / \mathrm{kg}$; doxorubicin at $2 \mathrm{mg} / \mathrm{kg}$ plus STF $50 \mathrm{mg} / \mathrm{kg}$ (STF was given 30 minutes before DOX administration). Tumor volumes were monitored during the experiment by a Vernier caliper (D0-D16) (A). After scarification (day 16) time, tumors were removed for measure tumor volume (B) and tumor weight (C). Changes in body weight during the experiment were recorded (D). Microphotographs of H\&E sections of internal organs from SCID-Beige mice after treatment with saline (control); STF alone at $50 \mathrm{mg} / \mathrm{kg}$; DOX alone at $2 \mathrm{mg} / \mathrm{kg}$; doxorubicin at $2 \mathrm{mg} / \mathrm{kg}$ plus STF $50 \mathrm{mg} / \mathrm{kg}$ (STF was given 30 minutes before DOX administration). Mice were sacrificed for pathologic examination 4 day after the last administration. Original magnification (x20) (E) 
(A)

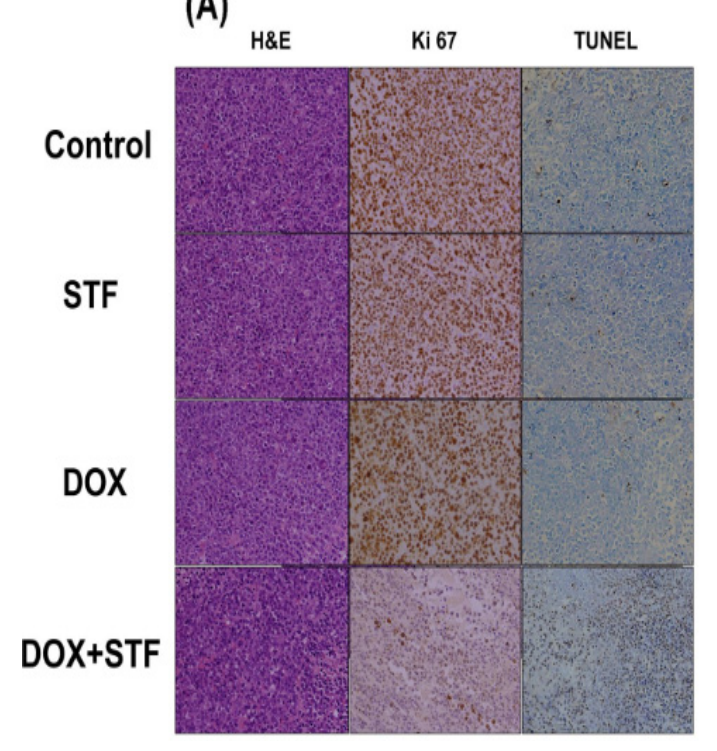

(B)

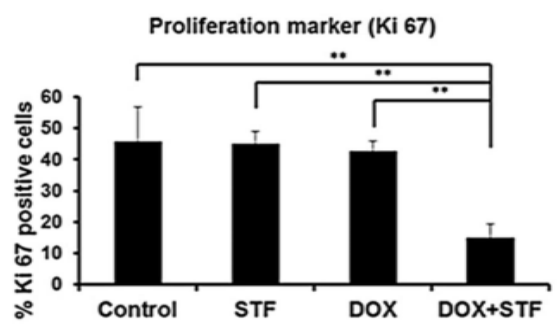

(C)

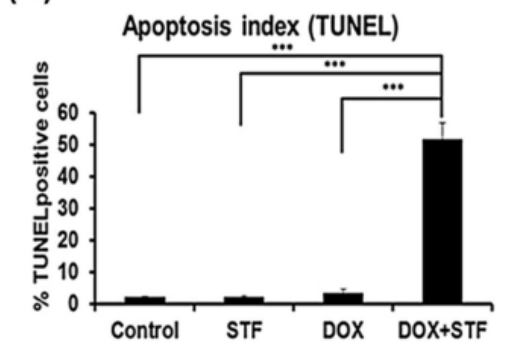

Figure 3. Tumor Masses were Cross-Sectioned and Fixed in 10\% Formalin Buffer and Prepared in Paraffin Embed Block. They were then stained with $\mathrm{H}$ and $\mathrm{E}$ and immunohistochemistry (A); (Ki67) proliferation marker and apoptotic marker (TUNEL). \% of proliferation marker (Ki67)-positive cells (B) and \% of apoptotic marker (TUNEL)-positive cells $(\mathrm{C})$ were analyzed using Image $\mathrm{J}$ software.

(A)

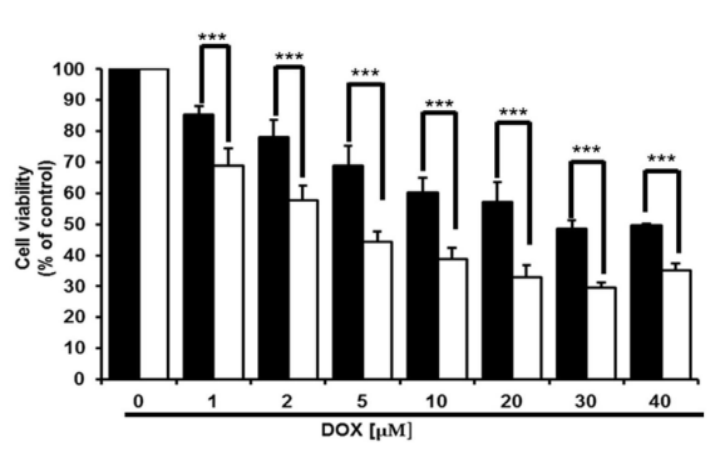

\section{K562/Adr}

DDOX

口DOX+STF

(B)

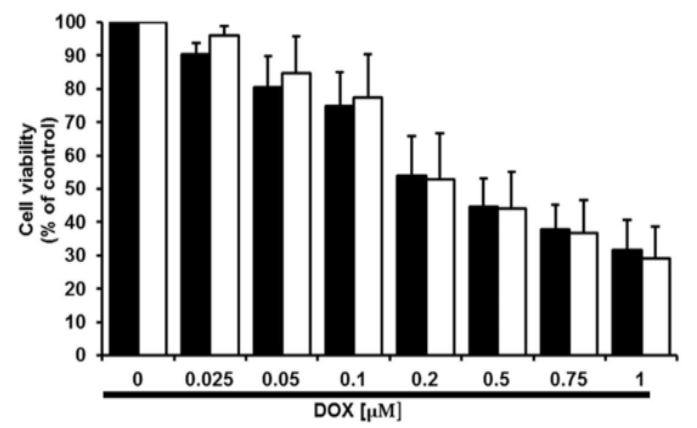

K562

$m$ DOX

QDOX+STF

(C)

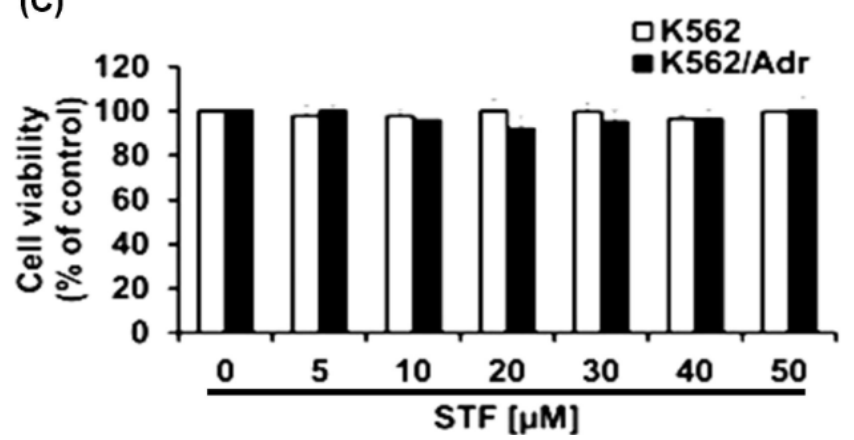

Figure 4. MDR Modulation Effect of STF on DOX Sensitivity in K562/Adr (A) and K562 Cell Lines (B) was determined. The co-administration of DOX $(0-40 \mu \mathrm{M})$ with or without STF was performed by MTT colorimetric assay for $48 \mathrm{hrs}$. The mean value from three independent experiments is shown and error bars indicating SD $(\mathrm{n}=3)$ are shown as $* * * p<0.001$ versus DOX single administration at each indicated concentration. 
(A)

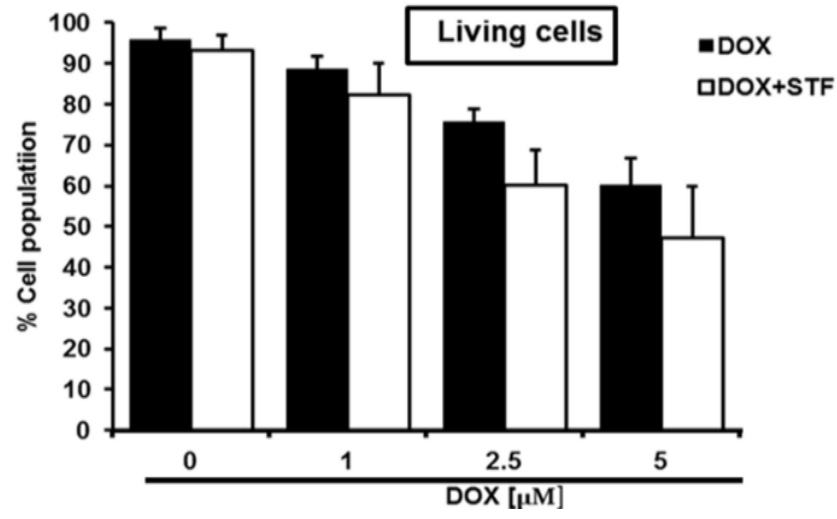

(B)

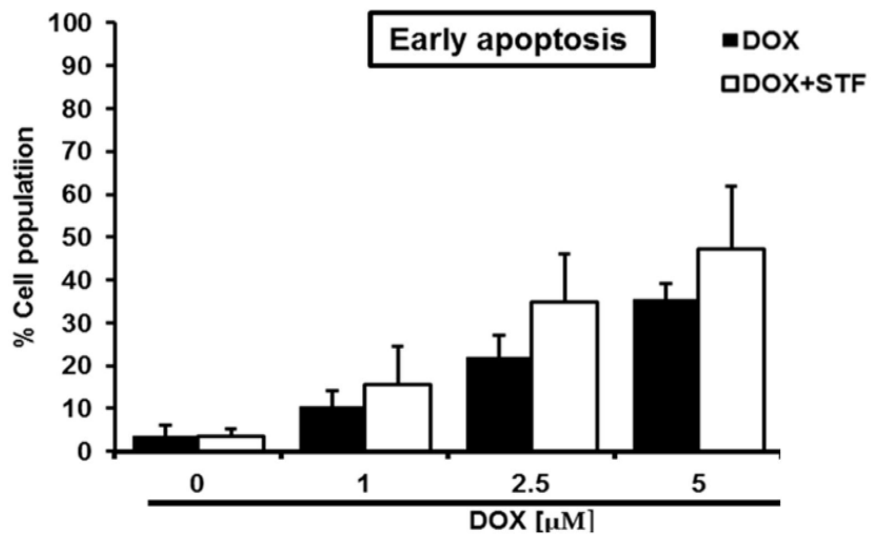

(C)

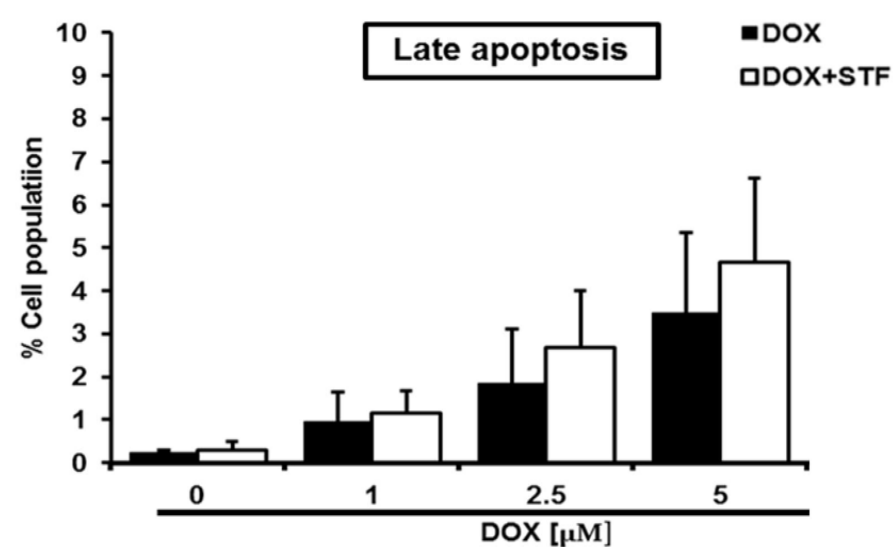

Figure 5. STF Enhanced Sensitivity of K562/Adr Cells to DOX. The graphs represent living cells (A), early apoptosis (B) and late apoptosis cell populations (C). K562/Adr cells were treated with various concentrations of DOX (0-5 $\mu \mathrm{M})$ with or without $5 \mu \mathrm{M}$ of STF for 48 hrs. After treatment, K562/Adr were washed twice with PBS and stained with annexin $\mathrm{V}$ and propidium iodide (PI). Apoptosis induction was determined by flow cytometry. The mean values from three independent experiments are shown and error bars indicate $\operatorname{SD}(n=3)$.

(Figure 4C). The results suggested that STF could enhance the sensitivity of the MDR leukemic cell line to DOX.

Enhanced sensitivity of MDR leukemic cells to chemotherapeutic drug by apoptosis and cell cycle arrest induction

Next, we investigated the effect of the STF and DOX co-administration induced program of cell death in K562/ Adr cells. The results indicated that co-administration of DOX and STF slightly revealed apoptosis induction in K562/Adr cells when compared to a single administration on both early and late apoptosis (Figure 5A-C). Next, cell-cycle determination in MDR leukemic cells was performed to confirm that the growth inhibition was due to the action of DOX. It was found that co-administration treatment of DOX and STF significantly induced G2/M phase arrest in K562/Adr cells at co-administration of DOX $1 \mu \mathrm{M}(p<0.05)$ and $5 \mu \mathrm{M}(p<0.001)$ when compared to the single administration (Figure 6A-C). The results suggested that the co-administration treatment of DOX and STF induced $\mathrm{G} 1$ and $\mathrm{G}_{2} / \mathrm{M}$ phase arrest. 
(A)

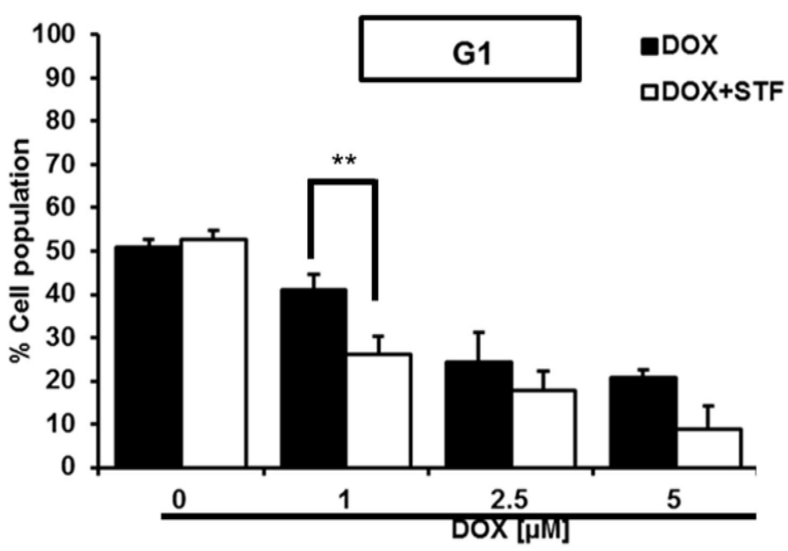

(B)

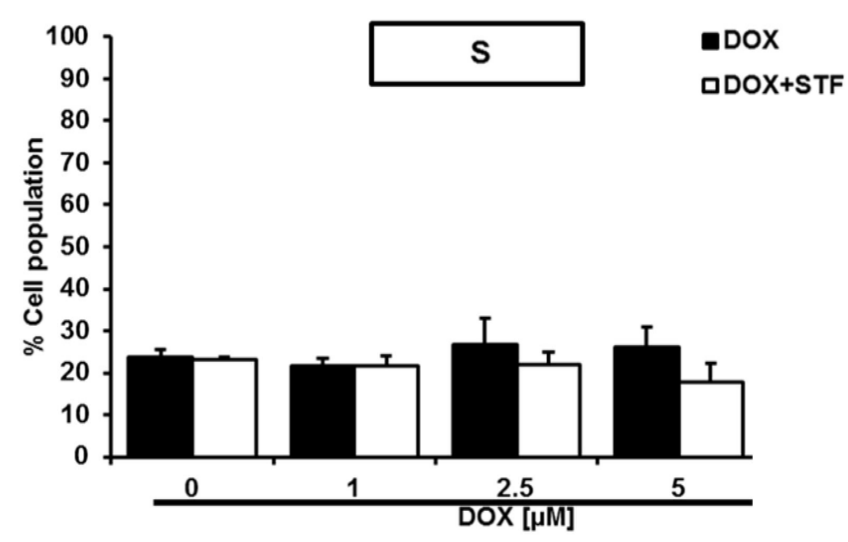

(C)

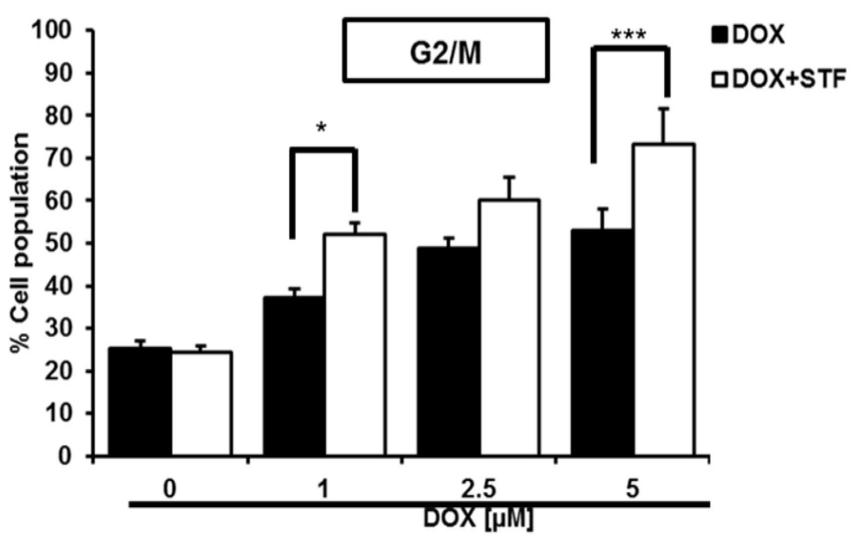

Figure 6. STF Enhanced Sensitivity of K562/Adr Cells to DOX. The graphs represent G1 (A), S (B) and G2/M phase distribution (C). K562/Adr cells were treated with various concentrations of DOX $(0-5 \mu \mathrm{M})$ with or without $5 \mu \mathrm{M}$ of STF for 48 hrs. After treatment, K562/Adr cells were washed twice with PBS and stained with propidium iodide (PI). Cell cycle phase distribution was determined by flow cytometry. The mean value from three independent experiments is shown and error bars indicate $\mathrm{SD}(\mathrm{n}=3) .{ }^{*} p<0.05, * * p<0.01$ and $* * * p<0.001$ versus DOX single administration at each indicated concentration.

\section{Discussion}

P-gp is a member of the transport protein superfamily called ATP-binding cassette (ABCB1/P-gp). It is a $170-\mathrm{kDa}$ transmembrane glycoprotein, which plays a major role in multidrug resistance (MDR) in various forms of cancer, such as in human cervical carcinoma cells, breast cancer adenocarcinoma cells and human leukemic cells (Acharyya et al., 2012). Moreover, it has a wide-spectrum substrate capacity, including for several chemotherapeutic drugs (anthracyclin, vinca alkaloids and paclitaxel) and fluorescence dyes (Calcein-AM and rhodamine 123). Therefore, P-gp can be the target for cancer treatment in order to enhance the efficacy of chemotherapeutic drugs in MDR cancer patients. Chemo-sensitizers or P-gp modulators are agents that abrogate the drug accumulation defect present in P-gp-expressing cancer cells. The magnitude of the chemo-sensitizer effects could be determined by comparing the $\mathrm{IC}_{50}$ values for a cytotoxic drug in the absence and presence of a relatively non- 
toxic, fixed concentration of the chemo-sensitizer (Marie et al., 1996). Verapamil (VER) and cyclosporine A (CSA) have been reported to be P-gp modulators in P-gp-mediated MDR cancer cells (Sharom et al., 1999; Krishna and Mayer, 2000), but they have limitations in clinical use due to their side effects (Ford and Hait, 1990). This has generated the need to search for new effective MDR modulators that have been isolated from natural products and that do not produce adverse side effects. A previous study reported that Stemona curtisii root extract, which contains Stemona alkaloids (Greger, 2006), modulated P-gp activity in human cervical cancer cells and the KB-V1 cell line (high P-gp expression). However, this was not the case with Multidrug resistant related protein-1 (MRP-1) activity in the MRP1-HEK293 cell line (high MRP-1expression). The results obtained from the present study strongly supported the finding that the Stemona plant extract may show a promising role as a P-gp modulator. In 2011, the MDR reversing property of three Stemona alkaloids (stemocurtisine; STC oxystemokerrine; OST and stemofoline; STF) isolated from Stemona burkillii (Chanmahasathien et al., 2011a) were studied and it was found that STF had the most potent effect on enhancing cancer drug sensitivity against human MDR cervical carcinoma cells (Chanmahasathien et al., 2011b). However, one limitation of natural compounds is that the variations of concentrations of the active compounds depend on the area of cultivation or the plant species. To solve this problem, semi-synthetic methods can increase the yields of active compounds and improve the compound efficacy by side chain modification of the parental core structure.

Stemona plants are comprised of three genus; Stemona, Croomia and Stichoneuron. The underground parts of the plant, roots and rhizomes have been used in Ayurvedic medicines with broad applications in Thailand, China and Japan. It has been reported that Stemona collinsiae root extract exhibited antineoplastic properties (Rungrojtrakool et al., 2012) and biological activity against human herpes virus (Akanitapichat et al., 2005). The investigation of the anti-cancer properties of Stemona alkaloids obtained from Stemonaceae holds significant promise for the development of more effective chemotherapeutic or adjuvant agents in cancer treatments. Previous studies point out the significant functional group that might regulate the P-gp modulating effect of Stemona alkaloids (Umsumarng et al., 2017). STF is a major alkaloid present in Stemona curtisii and Stemona burkillii, which is found in the southern and northern parts of Thailand, respectively (Kongkiatpaiboon et al., 2011). Additionally, DSTF is a major alkaloid of Stemona collinasae, which is found in the eastern part of Thailand (Kongkiatpaiboon et al., 2011). Recently, it has been reported that Stemona alkaloids isolated from Stemona curtisii could inhibit P-gp activity, whereas other alkaloids purified from Stemona javanica and Stichonueron halabalesis did not exhibit this property (Umsumarng et al., 2017). It could be concluded that alkaloid variations in the different Stemona species have an influence on their P-gp modulating activity. The present study supported the findings of previous studies that stated that the type of Stemona alkaloids included in Stemona plants can be used to indicate their chemosensitizing ability (Chanmahasathien et al., 2011a; Chanmahasathien et al., 2011b; Umsumarng et al., 2017). In the animal experiments, STF, a major alkaloid found in Stemona burkillii and Stemona curtisii that strongly exerts MDR reversal property, was selected to determine its chemosensitizing effect in vivo. Acute toxicity testing of STF in ICR mice was determined and it was found that all ICR mice could survive after i.p. injection of STF (50 mg/kg BW). Notably, there no lesions were found in the heart, lungs, liver, kidneys, or spleen as determined by gross and histopathology examinations. Furthermore, blood chemistry analysis showed that ALT, AST, BUN and creatinine levels were normal suggesting that the function of the liver and kidneys in the STF-treated mice was not altered. The results indicate that $50 \mathrm{mg} / \mathrm{kg} \mathrm{BW}$ of STF was found to be safe and can be utilized in further investigations. The P-gp modulating effect of STF was next evaluated in K562/Adr bearing SCID-beige mice. Twenty male SCID-beige mice were subcutaneously injected with K562/Adr cells in the flank area. The tumor size was measured every day. As soon as the tumor volume reached $100-200 \mathrm{~mm}^{3}$, the mice were divided into 4 groups: 1) vehicle control; $0.9 \% \mathrm{NaCl}$; I.P., 2) STF treatment (50 mg/kg BW; I.P.), 3) DOX treatment (2 mg/kg BW; IV) and 4) combination of DOX (2 mg/kg BW; IV) and STF (50 mg/kg BW; I.P.). After which, the treatment was initiated. Unfortunately, due to certain technical errors, tumor growth was observed in only in 12 of 20 transplanted-mice. Consequently, tumors were identified in three mice in each group. The co-administration of DOX and STF was found to clearly reduce tumor size when compared with the vehicle control group $(\mathrm{p}=0.3827)$, STF $(p=0.1371)$ and DOX $(p=0.4091)$. The survival rate of SCID-Beige mice was $100 \%$. The adverse effects on the body weight and internal organs of each group were not found to be different. Immunohistochemistry analyses found that the combined treatment significantly reduced the Ki67 proliferation marker $(\mathrm{p}<0.01)$ and induced apoptosis (TUNEL) $(\mathrm{p}<0.001)$ when compared to the DOX-treated group. Tumor growth did not differ in the single treatment of STF when compared to the control group. These results indicate that STF itself did not affect tumor growth, but STF was able to enhance the efficacy of DOX in K562/Adr-xenograft SCID-Beige mice. It can be concluded that STF could modulate P-gp-mediated MDR leading to an increased chemotherapeutic drug response in the animal model.

We have confirmed that STF and DOX co-administration induced program cell death by apoptosis analysis via flow cytometry. It was found that co-administration of DOX and STF initiated apoptosis induction in K562/Adr cells when compared to a single administration in both early and late apoptosis. The mechanism of DOX is a cell cycle non-specific agent. It could produce reactive oxygen species (ROS) and inhibit topoisomerase II leading to DNA damage and chromatin aggregation (Rabbani et al., 2005). However, in treatments of 48 hours, STF could inhibit P-gp function leading to increasing intracellular DOX accumulation. The DNA damage response mechanism of the cancer cells may be halted in some 
cell-cycle phases by repairing the DNA. However, if cancer cells were unable to repair DNA, programed cell death could be induced by apoptosis signaling.

Cell-cycle arrest in MDR leukemic cells was performed to confirm that the growth inhibition was due to the action of DOX by flow cytometry. It was found that the co-administration treatment of DOX and STF significantly induced G2/M phase arrest in K562/Adr cells through a co-administration of DOX $1 \mu \mathrm{M}(p<0.05)$ and 5 $\mu \mathrm{M}(p<0.001)$ when compared to a single administration. The results suggest that the co-administration treatment of DOX and STF induced $\mathrm{G}_{1}$ and $\mathrm{G}_{2} / \mathrm{M}$ phase arrest. Notably, DOX is the drug of choice to treat leukemic patients. DOX produces ROS and inhibits topoisomerase II leading to chromatin aggregation (Rabbani et al., 2005). It can arrest cancer cells in all cell-cycle phases and induces cell death. The results indicated that the DOX treatment caused cell accumulation at the G2/M phase, while the accumulation was shifted to sub-G1 and S populations when the cells were co-treated with STF. However, the cytotoxicity and growth inhibition might be due to the anti-cancer drugs that accumulated in the MDR cells and were stimulated by the STF. These results suggest that the compounds were able to enhance the drug sensitivity via the modulation of P-gp.

The present study provides scientific data on the biochemical mechanism of STF on P-gp overexpressing of MDR cancer cells. STF may be a candidate as a P-gp modulator as its toxicity is low and it produces no noticeable adverse effects. These compounds could be developed and used in combination with the conventional therapy of P-gp overexpressing in MDR cancer patients.

\section{Acknowledgements}

This work was supported by Chiang Mai University, Chiang Mai, Thailand, the National Research Council of Thailand (NRCT), and the Japan Student Services Organization (JASSO) Scholarship. The authors would like to thank Dr. Kittikorn Boonsri, Veterinarian Diagnostic Center, Faculty of Veterinary Medicine, Chiang Mai University, Chiang Mai, Thailand, for interpretation of all internal organs and tumor tissue slides and their pathological technique support, Dr. Kesuke Iida, Mrs. Miki Kanno and Mrs. Kaori Suzuki for their advice on flow cytometry and their valuable technical support. I would like to thank Dr. Yasuhito Kobayashi for his technical support in $\mathrm{H}$ and $\mathrm{E}$ and $\mathrm{Ki} 67$ on mice tumor tissue preparation and Professor Dr. Masami Suganuma for her technical support in all animal model experiments.

\section{References}

Acharyya S, Oskarsson T, Vanharanta S, et al (2012). A CXCL1 paracrine network links cancer chemoresistance and metastasis. Cell, 150, 165-78.

Akanitapichat P, Tongngok P, Wangmaneerat A, et al (2005). Antiviral and Anticancer Activities of Stemona collinsae. Thai J Pharm Sci, 29, 125-36.

Anuchapreeda S, Thanarattanakorn P, Sittipreechacharn S, et al (2006). Inhibitory effect of curcumin on MDR1 gene expression in patient leukemic cells. Arch Pharm Res, 29,
866-73.

Baruchel A, Leblanc T, Auclerc MF, et al (2009). Towards cure for all children with acute lymphoblastic leukemia?. Bull Acad Natl Med, 193, 1509-17.

Beider K, Darash-Yahana M, Blaier O, et al (2014). Combination of imatinib with CXCR4 antagonist BKT140 overcomes the protective effect of stroma and targets CML in vitro and in vivo. Mol Cancer Ther, 13, 1155-69.

Binkhathlan Z, Lavasanifar A (2013). P-glycoprotein inhibition as a therapeutic approach for overcoming multidrug resistance in cancer: current status and future perspectives. Curr Cancer Drug Targets, 13, 326-46.

Chanmahasathien W, Ampasavate C, Greger H, et al (2011a). Stemona alkaloids, from traditional Thai medicine, increase chemosensitivity via P-glycoprotein-mediated multidrug resistance. Phytomedicine, 18, 199-204.

Chanmahasathien W, Ohnuma S, Ambudkar S, et al (2011b). Biochemical mechanism of modulation of human P-glycoprotein by stemofoline. Planta Med, 77, 1990-95.

de Moraes A, Maranho C, Rauber G, et al (2013). Importance of detecting multidrug resistance proteins in acute leukemia prognosis and therapy. J Clin Lab Anal, 27, 62-71.

El-Readi M, Eid S, Ashour M, et al (2013). Modulation of multidrug resistance in cancer cells by chelidonine and Chelidonium majus alkaloids. Phytomedicine, 20, 282-94.

Fojo T, Menefee M (2007). Mechanisms of multidrug resistance: the potential role of microtubule-stabilizing agents. Ann Oncol, 18, 3-8.

Ford J, Hait W (1990). Pharmacology of drugs that alter multidrug resistance in cancer. Pharmacol Rev, 42, 155-99.

Gottesman M, Fojo T, Bates S (2002). Multidrug resistance in cancer : role of ATP-dependent transports. Nat Rev Cancer, 2, 8-58.

Greger H (2006). Structural relationships, distribution and biological activities of stemona alkaloids. Planta Med, 72, 99-113.

Hu J, Zhang N, Mao Y, et al (2009). Antitussive activity comparison of three kinds of Stemonae Radix in Chinese Pharmacopoeia. Zhongguo Zhong Yao Za Zhi, 34, 3096-104.

Kongkiatpaiboon S, Schinnerl J, Felsinger S, et al (2011). Structural relationships of stemona alkaloids: assessment of species-specific accumulation trends for exploiting their biological activities. $J$ Nat Prod, 74, 1931-8.

Krishna R, Mayer L (2000). Multidrug resistance (MDR) in cancer. Mechanism, reversal using modulators of MDR and the role of MDR modulators in influening the pharmacokinetics of anticancer drugs. Eur J Pharm Sci, 11, 265-85.

Lage H (2008). An overview of cancer multidrug resistance: a still unsolved problem. Cell Mol Life Sci, 65, 3145-67.

Lee H, Jung KH, Park S, et al (2014). Inhibitory effects of Stemona tuberosa on lung inflammation in a subacute cigarette smoke-induced mouse model. BMC Complement Altern Med, 14, 513.

Leonard G, Fojo T, Bates S, et al (2003). The role of ABC transporters in clinical practice. Oncologist, 8, 411-24.

Li Z, Sturm S, Stuppner H, et al (2007). The dichloromethane fraction of Stemona tuberosa Lour inhibits tumor cell growth and induces apoptosis of human medullary thyroid carcinoma cells. Biologics, 1, 455-63.

Lim D, Lee E, Jeong E, et al (2015). Stemona tuberosa prevented inflammation by suppressing the recruitment and the activation of macrophages in vivo and in vitro. $J$ Ethnopharmacol, 160, 41-51.

Limtrakul P, Chearwae W, Shukla S, et al (2007). Modulation of function of three $\mathrm{ABC}$ drug transporters, P-glycoprotein $(\mathrm{ABCB} 1)$, mitoxantrone resistance protein (ABCG2) 
and multidrug resistance protein 1 (ABCC1) by tetrahydrocurcumin, a major metabolite of curcumin. $\mathrm{Mol}$ Cell Biochem, 296, 85-95.

Marie J,Zhou D, Gurbuxani S, etal(1996). MDR1/P-glycoprotein in haematological neoplasms. Eur J Cancer, 32, 1034-8.

Matsumura I (2009). Standard therapies for acute myeloid leukemia. Rinsho Ketsueki, 50, 1389-400.

Mendizabal AM, Garcia-Gonzalez P, Levine PH (2013). Regional variations in age at diagnosis and overall survival among patients with chronic myeloid leukemia from low and middle income countries. Cancer Epidemiol, 37, 247-54.

Norgaard JM, Olesen LH, Hokland P (2004). Changing picture of cellular drug resistance in human leukemia. Crit Rev Oncol Hematol, 50, 39-49.

Pitchakarn P, Ohnuma S, Pintha K, et al (2012). Kuguacin $\mathrm{J}$ isolated from Momordica charantia leaves inhibits P-glycoprotein (ABCB1)-mediated multidrug resistance. J Nutr Biochem, 23, 76-84.

Punfa W, Suzuki S, Pitchakarn P, et al (2014). Curcumin-loaded PLGA nanoparticles conjugated with anti- P-glycoprotein antibody to overcome multidrug resistance. Asian Pac J Cancer Prev, 15, 9249-58.

Pyne SG, Jatisatienr A, Mungkornasawakul P, et al (2017). Phytochemical, synthetic and biological studies on stemona and stichoneuron plants and alkaloids: A personal perspective. Nat Prod Commun, 12, 1-6.

Quintas-Cardama A, Cortes JE (2006). Chronic myeloid leukemia: diagnosis and treatment. Mayo Clin Proc, 81, 973-88.

Rabbani A, Finn R, Ausio J (2005). The anthracycline antibiotics: antitumor drugs that alter chromatin structure. Bioessays, 27, 50-6.

Rogalska A, Koceva-Chyla A, Jozwiak Z (2008). Aclarubicin-induced ROS generation and collapse of mitochondrial membrane potential in human cancer cell lines. Chem Biol Interact, 176, 58-70.

Rungrojtrakool P, Siripong P, Yahuafai J, et al (2012). Antiproliferative activity against various cancer cells and phytochemical components of Thai herbal formula. Mahidol University Journal of Pharmaceutical Sciences, 2, pp7-14.

Sastraruji K, Sastraruji T, Pyne S, et al (2010). Semisynthesis and acetylcholinesterase inhibitory activity of stemofoline alkaloids and analogues. J Nat Prod, 73, 935-41.

Sastraruji K, Sastraruji T, Ung A, et al (2012). Synthesis of stemofoline analogues as acetylcholinesterase inhibitors Tetrahedron, 68, 7103-15.

Sharom FJ, Liu R, Romsicki Y, et al (1999). Insights into the structure and substrate interactions of the P-glycoprotein multidrug transporter from spectroscopic studies. Biochim Biophys Acta, 1461, 327-45.

Szwed M, Laroche-Clary A, Robert J, et al (2016). Efficacy of doxorubicin-transferrin conjugate in apoptosis induction in human leukemia cells through reactive oxygen species generation. Cell Oncol (Dordr), 39, 107-18.

Tiwari AK, Sodani K, Dai CL, et al (2011). Revisiting the ABCs of multidrug resistance in cancer chemotherapy. Curr Pharm Biotechnol, 12, 570-94.

Umsumarng S, Pintha K, Pitchakarn P, et al (2013). Inhibition of P-glycoprotein mediated multidrug resistance by stemofoline derivatives. Chem Pharm Bull, 61, 399-404.

Umsumarng S, Pitchakarn P, Sastraruji K, et al (2015). Reversal of human multi-drug resistance leukaemic cells by stemofoline derivatives via inhibition of P-glycoprotein function. Basic Clin Pharmacol Toxicol, 116, 390-7.

Umsumarng S, Pitchakarn P, Yodkeeree S, et al (2017). Modulation of P-glycoprotein by Stemona alkaloids in human multidrug resistance leukemic cells and structural relationships. Phytomedicine, 34, 182-90.

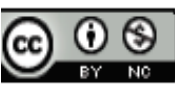

This work is licensed under a Creative Commons AttributionNon Commercial 4.0 International License. 